\title{
Serial discrimination reversal learning in pigeons as a function of intertrial interval and delay of reinforcement
}

\author{
Bertram O. Ploog \\ College of Staten Island, Staten Island, New York \\ and Graduate School and University Center, City University of New York, New York \\ AND \\ Ben A. Williams \\ University of California, San Diego, La Jolla, California
}

\begin{abstract}
Pigeons learned a series of reversals of a simultaneous red-green visual discrimination. Delay of reinforcement ( 0 vs. $2 \mathrm{sec})$ and intertrial interval (ITI; $4 \mathrm{vs.} 40 \mathrm{sec})$ were varied across blocks of reversals. Learning was faster with 0 -sec than with 2-sec delays for both ITI values and faster with 4-sec ITIs than with 40-sec ITIs for both delays. Improvement in learning across successive reversals was evident throughout the experiment, furthermore, even after more than 120 reversals. The potent effects of small differences in reinforcement delay provide evidence for associative accounts and appear to be incompatible with accounts of choice that attempt to encompass the effects of temporal parameters in terms of animals' timing of temporal intervals.
\end{abstract}

A large amount of previous work on serial reversal learning has shown that animals learn to reverse preference quickly in a simultaneous-choice procedure (e.g., Mackintosh, McGonigle, Holgate, \& Vanderver, 1968), sometimes to $100 \%$ accuracy after a single error (Dufort, Guttman, \& Kimble, 1954). The typical reversal procedure presents a simultaneous discrimination in which one choice alternative is designated as $S+$ (the stimulus that is correlated with reinforcement) and the other is designated as $S$ - (the stimulus that is correlated with extinction). Then, on successive reversals, the reward values of the two stimuli are reversed. The typical finding for serial reversal learning is that successive reversals are learned increasingly rapidly. Such effects have been described as "learning to learn," and they have been considered to be analogous to learning-set acquisition involving a large number of simple simultaneous discriminations.

Although much of the serial reversal literature has involved comparisons between species with respect to the rate at which learning improved over successive reversals (i.e., which animals exhibit the "learning to learn" phenomenon), various hypotheses have been offered for why learning rate improves across reversals. Unfortunately, the study of serial reversal learning was largely abandoned before a consensus was achieved about the underlying mechanisms.

A critical issue in the study of serial reversal learning, and learning to learn more generally, is whether the improvement in learning rate requires explanatory prin- ciples that are different from those for simple discrimination learning. For example, both may involve attentional competition between relevant and irrelevant stimuli, so that the irrelevant stimuli progressively lose salience over repeated reversals. Such an explanation would not rely on any principles for reversal learning that are not also involved in simple discriminations.

Despite the large number of experiments that have been conducted with the serial reversal procedure, surprisingly few researchers have studied learning parameters that might be diagnostic about the underlying process. One parameter that potentially has been studied is the time between successive trials: the intertrial interval (ITI). With longer ITIs, performance on serial reversal learning is substantially degraded, even after subjects have reached a high level of learning proficiency when trained initially with shorter ITIs (Williams, 1971, 1976). A finding of potential importance for understanding the facilitating effect of short ITIs in serial reversal learning is that a similar effect has not been found in studies of simultaneous discrimination learning in which a single discrimination problem has been presented (Biederman, 1967; see also Williams, 1977, for a discussion of an unpublished experiment). More recently, Williams (1998) studied the effects of ITI in a conditional discrimination task in which a tone or a light signaled whether the right or left lever, respectively, was correct. In that study, the rate of learning was increased with longer ITIs, an opposite effect from that seen in the earlier studies of serial reversal learning.

B. O. Ploog, bertram.ploog@csi.cuny.edu 
Williams (1998) also studied the effect of delay of reinforcement in the same simultaneous discrimination procedure; as expected, learning was substantially slower with longer delays. The effects of delay and ITI were independent and additive, moreover. Given that ITI appears to have opposite effects for simple discrimination versus serial reversal learning, the question is how delay of reinforcement and ITI will combine in serial reversal learning. The present study was thus an extension of the experimental design of Williams (1998) to serial reversal learning; that is, delay of reinforcement and ITI were varied, but in a serial reversal learning procedure in which the reinforcement contingencies were frequently reversed. Delay of reinforcement is itself of substantial interest, as its effects on serial reversal learning have not been studied previously.

In a preliminary experiment with pigeons, in which delays of $2 \mathrm{sec}$ and $6 \mathrm{sec}$ were compared, no improvement across successive reversals was evident. Consequently, the present study compared delays of $0 \mathrm{sec}$ and $2 \mathrm{sec}$. In another preliminary experiment using the same birds, 8-sec and 24-sec ITIs were compared, and no effect of ITI was found. In the present experiment, therefore, we chose 0 -sec and 2-sec reinforcement delays and 4-sec and 40 -sec ITIs.

\section{METHOD}

\section{Subjects}

Sixteen White Carneau pigeons served in this experiment. The birds were housed in individual cages under a 10h:14h dark:light cycle, with water and grit always available. They were maintained at $80 \%$ of their free-feeding weights with food (Purina, Gold Pellets) that was obtained during experimental sessions and with supplements in the home cages. All had served in a preliminary experiment that used a design that was similar but had a smaller difference in ITI values ( $8 \mathrm{sec}$ vs. $24 \mathrm{sec})$ and longer food-access times for reinforcement $(2.8 \mathrm{sec})$.

\section{Apparatus}

The experiment was carried out in four identical three-key standard pigeon conditioning chambers (Scientific Prototype, Model B 200 ) that were $42 \mathrm{~cm}$ high with a $38.0 \times 30.5 \mathrm{~cm}$ wire mesh floor (elevated by $7.0 \mathrm{~cm}$ ). The keys, which had a diameter of $3.2 \mathrm{~cm}$, were located $24.0 \mathrm{~cm}$ above the wire mesh floor. The keys were separated by $9.2 \mathrm{~cm}$, center to center. The keys could be illuminated by 12 -stimulus in-line projectors with $2.8-\mathrm{W}$ incandescent light bulbs (Type 1820X) and could be operated with a minimum force of $0.18 \mathrm{~N}$. Only red and green stimulus colors were used, and only the left and right keys were illuminated. The center key was not used in this experiment. The grain magazine (BRS/LVE, Model GFM-001) was accessible through a $6.0-\mathrm{cm}$ wide and $5.0-\mathrm{cm}$ high opening, which was located $5.5 \mathrm{~cm}$ above the wire mesh floor below the center key. When operated, the magazine access opening was illuminated by a 1.1-W incandescent lightbulb (Type 28PSB). The front wall (hinged on the bottom), the ceiling, and the back wall of each chamber were made of clear acrylic. The left and the right walls were made of bronze-colored 2-mm-thick sheet metal. The right wall served as the intelligence panel on which the keys, the stimulus projectors, and the hopper were mounted. The chambers were placed in sound-attenuated enclosures (Scientific Prototype, Model SPEC 2) that opened at the front. The enclosures had interior dimensions of $45.5 \times 62.5 \times 33.0 \mathrm{~cm}(\mathrm{~h} \times \mathrm{w} \times \mathrm{d})$. Each enclosure was equipped with a $0.3-\mathrm{W}$ loudspeaker $(7.5-\mathrm{cm}$ diameter) that was mounted in the far upper left corner to provide white noise that would mask extraneous sounds. Each enclosure was equipped with a fan to provide air circulation and additional masking noise. A 5-W incandescent lightbulb (Type FG 616) was mounted on the enclosure's ceiling, centered above the conditioning chamber. It served as a houselight, which was lit throughout each experimental session, including when the hopper was operated. The enclosures containing the conditioning chambers were located in a soundattenuated room, which was dark during the experimental sessions. The experimental room was adjacent to the room that contained the computer (Apple Macintosh G3 "Blue \& White Minitower"), which controlled the experimental events and performed data collection. The interface consisted of a multipurpose I/O card (National Instruments, PCI-DIO-96) and optorelays to read keypecks and to control stimulus presentations. The software was custom-written in $\mathrm{C} / \mathrm{C}++$ (Metrowerks, CodeWarrior 9.0).

\section{Procedure}

Given that the birds had prior experience, no pretraining was needed. The birds were simply started with training on a simultaneous-discrimination paradigm. The houselight was lit throughout the entire session. A session started with an ITI (4 sec or $40 \mathrm{sec}$ ). After the ITI, the left and right keys were illuminated with a red and a green stimulus. Pecks to one stimulus $(\mathrm{S}+)$ resulted in termination of both keylights and led to a brief reinforcement delay $(0 \mathrm{sec}$ or $2 \mathrm{sec})$ before $2 \mathrm{sec}$ of access to grain was provided. After reinforcement, the next ITI was initiated. Pecks to the alternative stimulus $(\mathrm{S}-$ ) also resulted in termination of both keylights and led to the same brief delay, but no reinforcement was provided. Instead, the next ITI started immediately after the delay. The stimulus situation during the delays and the ITI was identical (i.e., houselight was lit, but all keys were dark). A total of five pecks to either S+ or $\mathrm{S}-$ (counted separately) was required to initiate the delay of reinforcement. The positions of S + and $\mathrm{S}-$ were random except for the following restrictions: (1) A correction procedure was used after $\mathrm{S}-$ choices, so that the positions of the colors remained the same on the next trial; (2) the positions never remained the same for more than three consecutive trials, unless the correction procedure was in effect; and (3) each stimulus was presented on both sides equally often, not including the correction trials. Each session was in effect for 80 trials (ITI and stimulus-presentation sequence).

Conditions differed by the combination of ITI and delay values; thus, there were four conditions, labeled condition $4 / 0$, condition $4 / 2$, condition $40 / 0$, or condition $40 / 2$, with the notation ITI/ delay in seconds. A condition began with 2 sessions with one color/ trial-outcome correlation (e.g., red/S+, green/S-). After 2 sessions with the original color/trial-outcome correlation, the correlation was reversed. From that point on, a reversal occurred after every 2 sessions, resulting in alternation between the original and the reversed correlation every other session. Each condition continued until 20 reversals (40 sessions) were completed.

Eight birds were trained with the 0 -sec delay conditions, and the remaining 8 birds received the 2-sec delay throughout. Each delay group received three phases of training (each phase lasted for 40 sessions-i.e., for 20 two-session reversals). During Phase 1, half of each group's subjects received the 4-sec ITI and half received the 40 -sec ITI (original ITI). In Phase 2 (reversed ITI), each bird was switched to the alternative ITI value, but the delay value remained the same (e.g., from $40 / 0$ to $4 / 0$ or from $4 / 2$ to $40 / 2$ ). In Phase 3 (replicated original ITI), each bird was switched back to its original ITI value, but the delay value remained the same. This constituted a complete ABA design that tested for the effects of ITI as a within-subjects factor.

\section{RESULTS}

Preliminary analyses of the results in terms of proportion correct $(p)$ indicated that ceiling effects affected the analysis of the interactions between delay and ITI. We thus chose as a dependent variable logit $p$ - that is, $\log _{10}[p /(1-p)]$ - 
because this measure does not limit the data to a ceiling or to a floor (cf. Williams, 1998).

It should be noted that all subjects had extensive prior experience on reversal learning with the same stimuli, but with smaller differences in the ITI values ( 8 vs. $24 \mathrm{sec}$, rather than the current 4 vs. $40 \mathrm{sec}$ ). The earlier study used the 0 - and 2-sec delays that were studied here. Birds with a prior history of 0 -sec delay were assigned to the 2-sec delay condition and vice versa. Because of this extensive prior training, it was important to determine whether improvement in reversal learning was continuing to occur. Figure 1 provides a comparison of the overall level of discrimination proficiency during Phase 1 and during Phase 3, when the birds had the same ITI and delay values (i.e., Phase 3 was an exact replication of Phase 1, and the two were separated by Phase 2, which is not shown in Figure 1). Correction trials were included, because these trials constituted additional training trials. The data are plotted as a function of five blocks of four reversals, ITI duration, reinforcement delay, and session of reversal. The results that are shown in Figure 1 were analyzed using an ANOVA, with delay ( 0 vs. 2 sec) and ITI ( 4 vs. $40 \mathrm{sec}$ ) as between-subjects factors and phase (Phase 1 vs. Phase 3), block (five), and session (Session 1 vs. Session 2) as within-subjects factors. Overall, performance was higher in Phase $3(M=0.69, S E=0.08)$ than in Phase $1(M=0.54, S E=0.05)[F(1,12)=11.3, p<$ $.010]$. For the top two panels in Figure 1, which show the results for Session 1, there was a higher level of discrimination in Phase 3 for all four conditions, although the differences were small. Session 2 of training, shown in the bottom panels, had an even smaller difference between conditions. This phase $\times$ session interaction was confirmed by the ANOVA $[F(1,12)=12.2, p<.005]$. The phase $\times$ delay interaction was also significant $[F(1,12)=$ $5.8, p<.050]$. In order to explore this interaction in more detail, the analysis was conducted separately for the two delays (with Bonferroni corrections; significance level set at $\alpha=.025)$. Phase was significant for the 0 -sec delay for Phase $1(M=0.77, S E=0.07)$ and for Phase $3(M=1.03$, $S E=0.11)[F(1,6)=19.2, p<.010]$, but it was not significant for the 2-sec delay for Phase $1(M=0.31, S E=$ $0.07)$ or for Phase $3(M=0.36, S E=0.11)[F(1,6)<1.0$, $p=.545]$. With delay included in the analysis, none of the other interactions involving phase were significant. The main effect of ITI was marginally not significant for both

Original ITI (Phase 1)

\section{Replicated Orig. ITI (Phase 3)}

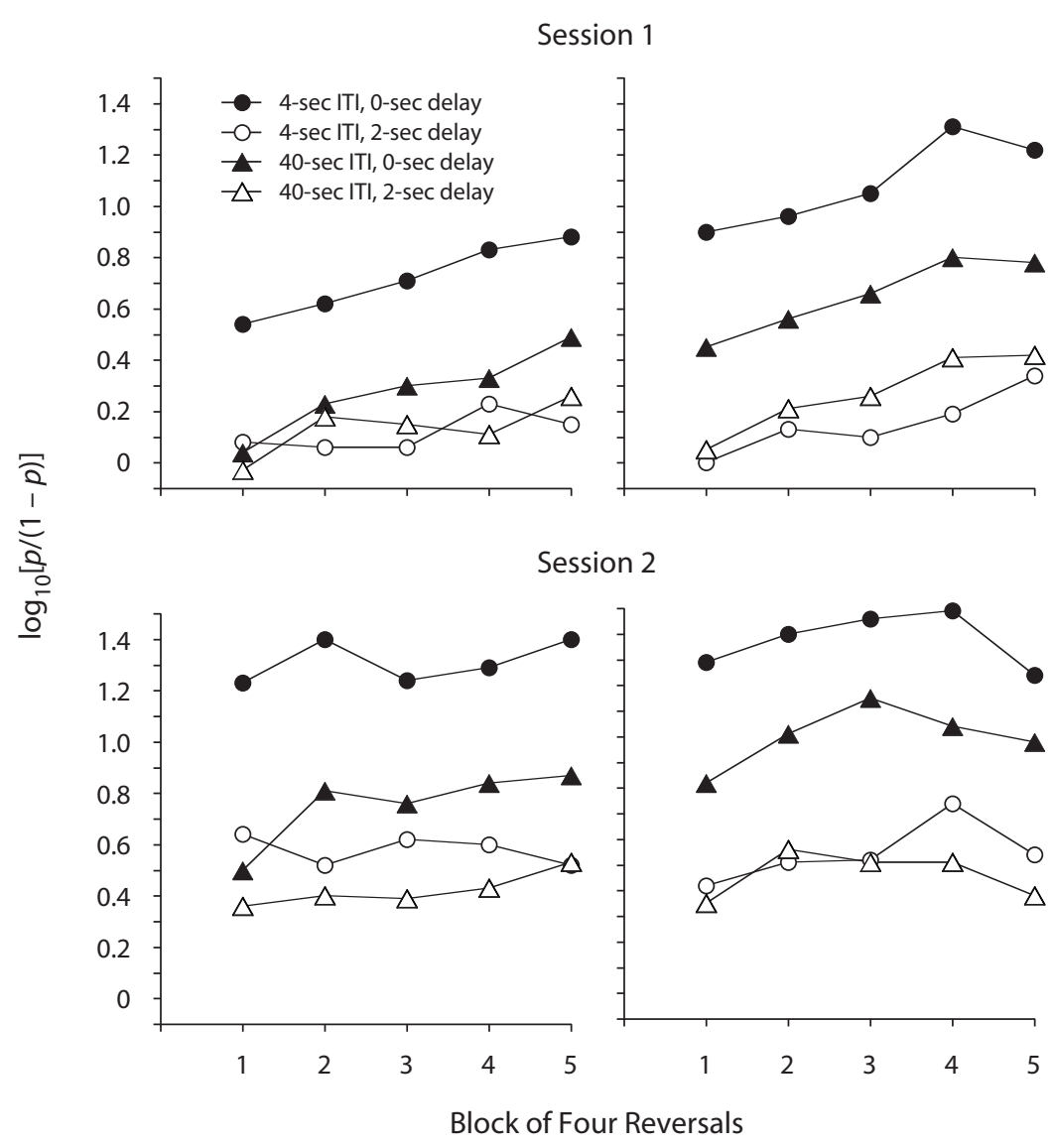

Figure 1. The "learning to learn" effect across Phases 1 and 3: means $(N=4)$ of $\log _{10}[p /(1-p)]$ as a function of block of four reversals, ITI, delay, and session within reversal. Each symbol tracks an individual bird across Phases 1 and 3. 
the 4-sec ITI $(M=0.74, S E=0.09)$ and the 40-sec ITI $(M=0.50, S E=0.09)[F(1,12)=3.8, p=.075]$, whereas the main effect of delay was significant $[F(1,12)=21.2$, $p<.005]$. The ITI $\times$ delay interaction was not significant $[F(1,12)=3.0, p=.106]$. Finally, the main effects of block $[F(4,48)=13.3, p<.001]$, and session $[F(1,12)=$ 93.8, $p<.001]$ were significant, as was the block $\times$ session interaction $[F(4,48)=7.4, p<.001]$, as can be seen in Figure 1. That is, the increase over blocks was greater for Session 1 than for Session 2, and performance was lower in Session 1 than in Session 2.

Figure 2 shows logit $p$ as a function of the number of reversals. The means were obtained by first averaging the data of Phases 1 and 3, and then combining those data with the data of Phase 2, so that all subjects - in order to do a within-subjects analysis - had data for both ITI values. The results that are shown in Figure 2 were analyzed using an ANOVA with delay and order (whether a bird received the 4- or 40-sec ITI in Phase 1 and Phase 3) as between-subjects factors, and ITI, block of four reversals, and session as within-subjects factors. Because order was not significant $[F(1,12)=0.5, p=.481]$, it was dropped, and the analysis was rerun. The 0 -sec delays (circles) produced better performance than did the 2-sec delays (triangles) $[F(1,14)=27.6, p<.001]$, and 4-sec ITIs (filled symbols) produced better performance than did 40-sec ITIs (open symbols) $[F(1,14)=12.3, p<$ $.005]$. Even though the delay $\times$ ITI interaction was not significant $[F(1,14)<1.0, p=.380]$, it was explored in greater detail - because of its potential importance for the present article - by conducting the analysis separately for the two delays (with Bonferroni corrections; significance

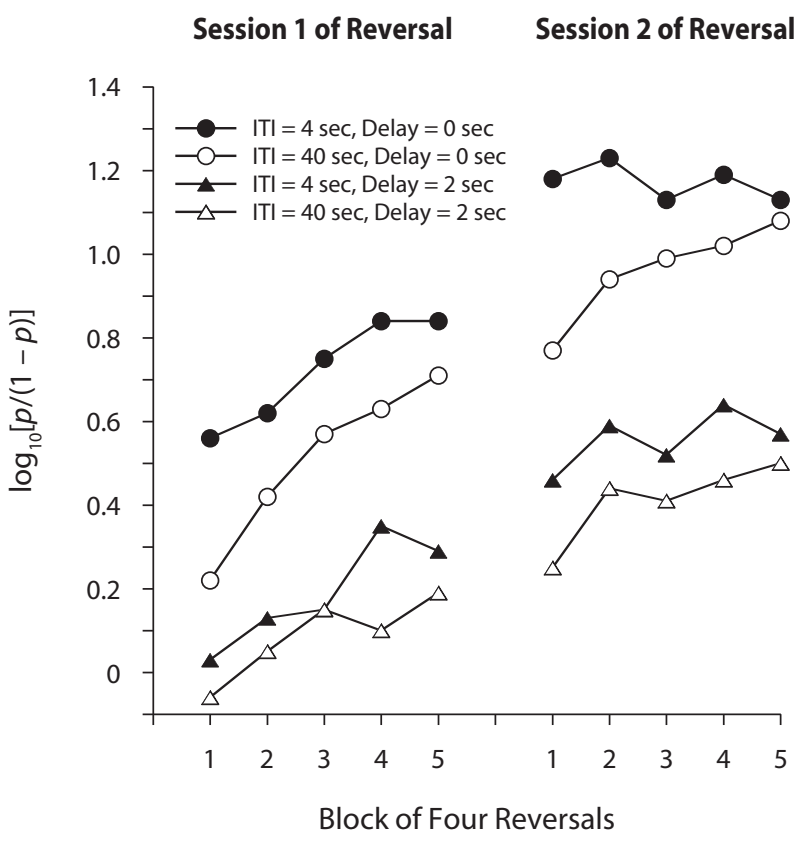

Figure 2. ITI and delay effects collapsed over Phases 1, 2, and 3: means $(N=4)$ of $\log _{10}[p /(1-p)]$ over Phases 1,2 , and 3 as a function of block of four reversals, ITI, delay, and session within reversal.

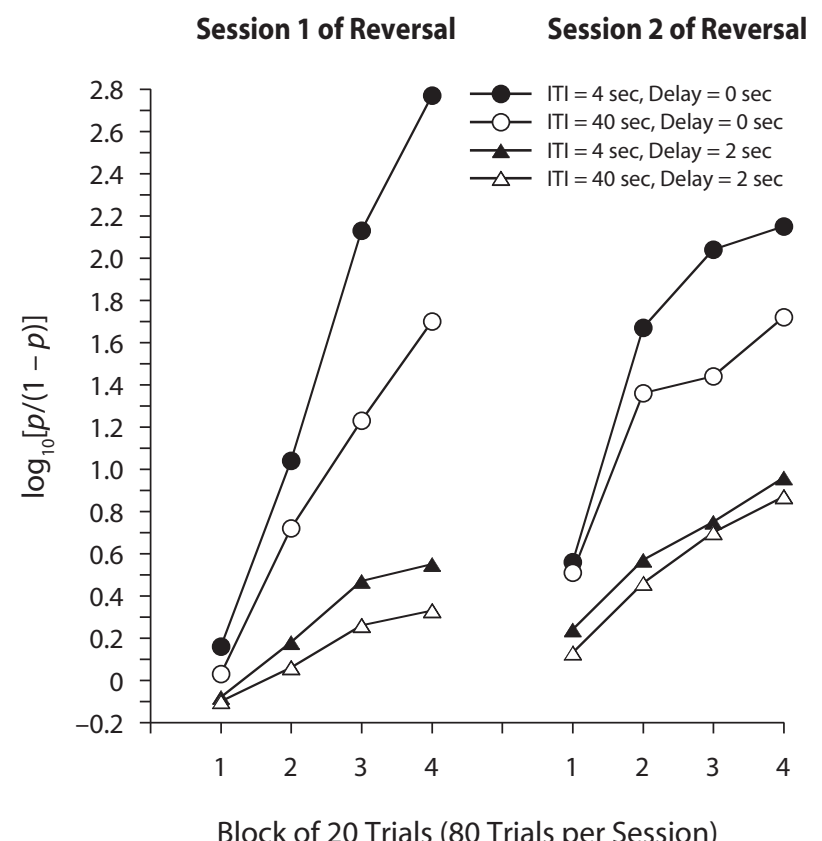

Figure 3. ITI and delay within-session effects: means $(N=4)$ of $\log _{10}[p /(1-p)]$ of the last 10 reversals of Phases 1,2 , and 3 as a function of 20-trial block (one quarter of the session), ITI, delay, and session within reversal.

level set at $\alpha=.025)$. For the 0 -sec delay, ITI yielded $F(1,7)=7.9, p=.026$, whereas for the 2-sec delay, ITI yielded $F(1,7)=4.4, p=.074$.

Continuing with the full analysis (delay included), the 4-sec and 40-sec ITI conditions appeared to produce converging performance overall across blocks for both sessions, indicating an ITI $\times$ block interaction $[F(4,56)=$ 3.0, $p<.050]$. All other interactions involving delay and ITI were not significant. Overall performance in the first session of the reversal was lower than was performance in the second session $[F(1,14)=189.3, p<.001]$. There was a consistent improvement across block (reversals) in both sessions $[F(4,56)=25.6, p<.001]$. Finally, the improvement across reversals was more pronounced in Session 1 than it was in Session 2, indicating a block $\times$ session interaction $[F(4,56)=8.0, p<.001]$.

Figure 3 depicts the course of the within-session acquisition across the two sessions of training on each reversal. Specifically, it shows performance across blocks of 20 trials (one quarter of the sessions) for both sessions of the reversals. As in Figure 2, the data of Phases 1 and 3 were averaged first, then the resulting data were combined with the data of Phase 2 in order to do a within-subjects comparison of the ITI effects. Because our interest for this analysis was mainly in stable behavior, we included the data of only the last half of each phase by collapsing over the last 10 of 20 reversals for each phase. The results that are shown in Figure 3 were analyzed using an ANOVA with delay as a between-subjects factor and ITI, session, and block as within-subjects factors. Figure 3 shows that 0 -sec delays (circles) produced better performance than did 2-sec delays (triangles) $[F(1,14)=26.2, p<.001]$ and that 4 -sec 
ITIs (filled symbols) produced better performance than did 40 -sec ITIs (open symbols) $[F(1,14)=14.3, p<.005]$. The ITI effect was larger for the 0 -sec delay (circles) than for the 2 -sec delay (triangles); thus, there was a significant delay $\times$ ITI interaction $[F(1,14)=5.3, p<.050]$. As in Figure 2, the analysis was rerun for delay separately (with Bonferroni corrections; significance level set at $\alpha=.025$ ). For the 0 -sec delay, ITI yielded $F(1,7)=14.1, p=.007$, whereas for the 2-sec delay, ITI yielded $F(1,7)=1.6, p=.250$.

Continuing with the full analysis (delay included), it was confirmed that the effect of delay was larger for the last quarter of a session than for the first (i.e., the functions with circles and triangles diverged from the beginning of Sessions 1 and 2 toward the end of each session), a result that was validated by a significant delay $\times$ block interaction $[F(3,42)=13.2, p<.001]$. Similarly, the effect of ITI was larger for the last quarter of a session than for the first (i.e., the functions for the same symbol diverged from the beginning of Sessions 1 and 2 toward the end of each session), a result that was validated by a significant ITI $\times$ block interaction $[F(3,42)=5.7, p<.005]$. As can also be seen in Figure 3, the degree of divergence of lines with the same symbol depended on delay-circles or triangles - and on block. Indeed, the delay $\times$ ITI $\times$ block interaction was significant $[F(3,42)=3.7, p<.050]$. The remaining significant effects were session, with higher performance in Session $2(M=1.01, S E=0.08)$ than in Session $1(M=0.72, S E=0.11)[F(1,14)=32.4, p<$ $.001]$; block, with higher performance toward the end of a session) $[F(3,42)=59.9, p<.001]$; session $\times$ block, with a stronger effect of block for Session 1 than for Session $2[F(3,42)=7.5, p<.001]$; and session $\times$ block $\times$ delay $[F(3,42)=11.7, p<.001]$. The triple interaction is apparent in Figure 3 from the greater degree of divergence of the pairs of lines (with the same symbols) in Session 1 than in Session 2.

An additional observation in Figure 3 is of substantial interest: The performance in the first 20 trials of the second session of training was substantially reduced from the level that was obtained at the end of the first session. This can be seen in Figure 3, in the comparison of the same symbols of Block 4 data in the left set of functions (Session 1) with Block 1 data in the right set of functions (Session 2). This effect was apparent for all four conditions. Such a pattern has been seen in previous studies of reversal learning, and it has been interpreted as the result of subjects' forgetting what was learned in Session 1 during the $24 \mathrm{~h}$ that separated the two training sessions (Woodward, Schoel, \& Bitterman, 1971), or as the result of spontaneous recovery of the preferences that were established in previous training sessions (Mazur, 1995, 1996).

\section{DISCUSSION}

The results demonstrate that there was continued improvement in the learning rate across successive reversals, even after a long history of repeated reversals; however, this improvement (Phase 1 vs. Phase 3 ) was substantially greater for the 0 -sec delay groups $(M=0.77$ vs. $M=$ $1.03)$ than for the 2 -sec delay groups $(M=0.31$ vs. $M=$
$0.36)$. "Learning to learn" is thus affected by delay of reinforcement, just as is simple discrimination learning, which is a novel finding. Note that this result was obtained despite extended previous exposure of the birds to a similar procedure (with different ITI values).

The ITI effects were consistent with those that have been observed in previous studies of ITI in serial reversal learning (Williams, 1971, 1976), since reversal learning in the earlier experiments was more proficient with shorter ITIs. The size of the ITI effect that was seen here seems considerably less robust than that obtained in previous studies, however. Indeed, there was considerable variability in the ITI effects across subjects, which necessitated a within-subjects design in order for the ITI effect to be clearly detected. Even with the within-subjects statistical analysis, the effects of ITI were statistically significant for only the 0 -sec delay condition, although the nonsignificant differences that were obtained with the 2 -sec delay were in the same direction of better learning with shorter ITIs.

In previous studies of very similar color-discrimination procedures - which also used within-subjects comparisons, always with zero delay of reinforcement - the number of errors emitted in reaching a fixed criterion of 10 consecutively correct trials varied from 4 per reversal with a 4-sec ITI, to 10 per reversal with a 30-sec ITI, to 21 per reversal with a 60 -sec ITI (Williams, 1971). A possible explanation for the smaller ITI effect that was seen in the present study is that we used a fixed amount of training per reversal (two sessions), whereas prior work trained subjects to a fixed criterion of 10 consecutively correct choices. The use of this performance-based criterion in prior studies likely had the effect of amplifying initially small differences in learning rate. For example, if a subject was $80 \%$ correct with a long ITI, it could have taken many more trials to reach the 10 consecutively correct criterion than if a subject was $90 \%$ correct with a short ITI. That is, the differences in the number of trials to criterion was not necessarily proportional to the differences in the percentage of correct choices, because each error caused the subject to revert to the start of the run of the required 10 consecutively correct trials before the learning of a given reversal was terminated.

The faster learning that was seen with the shorter ITIs in the present study is opposite to the effect of ITI that was reported by Williams (1998) in his study of the acquisition of a simple conditional discrimination (e.g., a light signaled that the left lever was correct, and a noise signaled that the right lever was correct). One possible explanation for this difference is that "learning to learn" procedures, such as serial reversal learning, involve different or additional processes when compared with simple discriminations (cf. Bessemer \& Stollnitz, 1971). For example, Williams (1976) proposed that the basis of the ITI effect in serial reversal learning was that subjects learned to use the outcome from the preceding trial as a conditional cue that was stored in short-term memory and that was therefore susceptible to decay over longer retention periods (i.e., ITI).

But numerous other differences in both subjects and procedure could also explain the opposite ITI effects that 
were seen in the present study versus those that were observed by Williams (1998). Williams (1998) used rats as subjects, whereas the present study used pigeons. Also, Williams (1998) arranged a conditional discrimination in which only one discriminative stimulus (light vs. noise) was presented on a given trial (cuing whether the right or left lever was correct), whereas both choice stimuli were simultaneously available in the present study. It is possible that long ITIs enhance the signal value of a stimulus that is presented alone, whereas any such signal enhancement would affect both stimuli $(\mathrm{S}+$ and $\mathrm{S}-$ ) of a simultaneous discrimination.

An important issue is how, if at all, the ITI and delay variables interacted. Whereas Williams (1998) found the effects of these variables to be independent and additive, previous research involving both autoshaping (Gibbon, Baldock, Locurto, Gold, \& Terrace, 1977) and delayed matching to sample (Roberts \& Kraemer, 1982) reported significant interactions between delay and ITI, since the controlling variable was their ratio and not their absolute values. In the present study, only one of the three ANOVAs (for Figure 3) reported a significant delay $\times$ ITI interaction (the other two analyses had nonsignificant effects in the same direction), but the interaction suggested that ITI effects were larger with the 0 -sec delay condition - that is, the opposite effect of the ITI-to-delay ratio.

Perhaps the present results are an artifact of the specific ITI-to-delay ratios that were chosen. This seems unlikely, however, in light of our preliminary studies - data that are not reported here-which used, in part, the same birds that served in the present experiment. Specifically, the delay/ITI ratios $0 / 4,0 / 16,0 / 24,0 / 40,2 / 4,2 / 8,2 / 24,2 / 40$, $6 / 8$, and $6 / 24$ did not yield a pattern that suggested that the results were dependent on the ratio.

The largest effect in the present experiment was the difference in learning rate between the 0 -sec delay condition and the 2-sec delay condition. For example, discrimination accuracy at the end of the first session of the reversals was an order of magnitude greater with the 0 -sec than with the 2-sec delay (see Figure 3). Recognizing the power of the delay-of-reinforcement parameter-which has been firmly established in many previous studies - is important in this context, because much recent discussion has considered reinforcement delay to be an incentive parameter that is fundamentally similar to other reinforcement parameters (e.g., amount) in determining reinforcement value (Kyonka \& Grace, 2008). Moreover, the effect of reinforcement delay in concurrent chains, in which the delays correspond to the durations of the terminal links of the chains, has been interpreted as the result of subjects "timing" the relative duration of the terminal links and then choosing on the basis of an all-or-none decision process in favor of the shorter delay (Kyonka \& Grace, 2007). Such a view of reinforcement delay seems unlikely to encompass the increase in discrimination difficulty that was caused by the brief delays that were studied here.

It should be recognized, however, that duration of the terminal links in a concurrent-chains schedule is critically different from the delay of reinforcement that was manipulated in the present study. Namely, the terminal- link stimuli in concurrent chains - which are correlated with the different delays - are conditioned reinforcers that are immediately contingent on the choice responses, and it is the value of those conditioned reinforcers that is determined by the times to reinforcement that are signaled by their onsets. The delays that were used here were unsignaled, so that a temporal gap separated the choice response and its outcome - a temporal gap that greatly retarded the acquisition of the discrimination. The most plausible interpretation of this deficit is that the temporal gap impeded the associative connection between the choice responses and their consequences.

Several studies of choice in concurrent schedules (Dreyfus, 1991; Gallistel, Mark, King, \& Latham, 2001; Grace, Bragason, \& McLean, 2003; Grace \& McLean, 2006; Mark \& Gallistel, 1994; Mazur, 1997; Schofield \& Davison, 1997) have reported that both rats and pigeons may reverse their preferences quickly when the reinforcement values that are contingent on the choice alternatives are reversed, either between successive sessions or within sessions. This rapid reversal of preference is strikingly different from the gradual change in preference that has been noted in the great majority of concurrent-schedule experiments, most of which involve training with a given pair of schedule values for multiple sessions (e.g., 15-30) until preference reaches a stability criterion. Such rapid improvement in learning with frequent reversals in the contingencies is parallel to the improvement that typically occurs in serial reversal learning. If this similarity is more than superficial, the implication is that the increasing rate of learning each new reversed contingency in a concurrent schedule should be sensitive to ITI and to delay of reinforcement in a manner that is similar to that seen here.

Kyonka and Grace (2009) have reported results from a concurrent-chains procedure that are analogous to those that are reported here, although instead of varying the ITI, they varied the duration of the initial link of the schedule. Pigeons chose between fixed interval (FI) 10 -sec versus FI 20-sec terminal-link schedules, with the choice alternative that was assigned the shorter terminal link reversed between sessions. Initial-link duration was also varied randomly between sessions. Both the terminal level of preference at the end of a session and the rate of preference increase were greater for the shorter initial links. Whether this parallel is more than superficial remains to be determined.

At least procedurally, the delays of reinforcement that were studied here are analogous to the terminal-link durations of a concurrent chain, but only if the same stimulus occurs during both terminal links (see, e.g., Ploog, 2001; Williams \& Fantino, 1978). Such schedules have been studied but never, to our knowledge, with repeated reversals of the schedule contingencies. Williams and Fantino did report extreme difficulty in single preference reversals with nondifferential stimuli in the terminal links, especially when longer terminal links were used, an effect that is possibly analogous to the effect that was seen here.

Although the serial-reversal problem that was studied here may not be readily susceptible to a timing interpretation, it remains to be seen whether repeated reversals in a 
concurrent-chain procedure are controlled by the same or by different principles.

\section{AUTHOR NOTE}

This research was supported in part by a PSC-CUNY Grant to the first author and by funds for animal maintenance that were provided by the College of Staten Island Divisional Dean and by the Department of Psychology. This paper is dedicated in memoriam to Anita Conte, Facilities Manager of the social and natural sciences buildings at CSI, who with her dedication and service to the college over many years made it possible for us to conduct research humanely, ethically, and efficiently. Correspondence concerning this article should be sent to Bertram O. Ploog, Dept. of Psychology, 4S-105, College of Staten Island, City University of New York, 2800 Victory Blvd., Staten Island, NY 10314 (e-mail: bertram.ploog@csi.cuny.edu).

\section{REFERENCES}

Bessemer, D. W., \& Stollnitz, F. (1971). Retention of discriminations and an analysis of learning set. In A. M. Schrier \& F. Stollnitz (Eds.), Behavior of nonhuman primates: Modern research trends (Vol. 4, pp. 1-58). New York: Academic Press.

Biederman, G. B. (1967). Simultaneous discrimination: Parameters of reinforcement and ITI. Psychonomic Science, 8, 215-216.

Dreyfus, L. R. (1991). Local shifts in relative reinforcement rate and time allocation on concurrent schedules. Journal of Experimental Psychology: Animal Behavior Processes, 17, 486-502.

Dufort, R. H., Guttman, N., \& Kimble, G. A. (1954). One-trial discrimination reversal in the white rat. Journal of Comparative \& Physiological Psychology, 47, 248-249.

Gallistel, C. R., Mark, T. A., King, A. P., \& Latham, P. E. (2001). The rat approximates an ideal detector of changes in rates of reward: Implications for the law of effect. Journal of Experimental Psychology: Animal Behavior Processes, 27, 354-372.

Gibbon, J., Baldock, M. D., Locurto, C., Gold, L., \& Terrace, H. S. (1977). Trial and intertrial durations in autoshaping. Journal of Experimental Psychology: Animal Behavior Processes, 3, 264-284.

Grace, R. C., Bragason, O., \& McLean, A. P. (2003). Rapid acquisition of preference in concurrent chains. Journal of the Experimental Analysis of Behavior, 80, 235-252.

Grace, R. C., \& McLean, A. P. (2006). Rapid acquisition in concurrent chains: Evidence for a decision model. Journal of the Experimental Analysis of Behavior, 85, 181-202.

Kyonka, E. G. E., \& GRACE, R. C. (2007). Rapid acquisition of choice and timing in pigeons. Journal of Experimental Psychology: Animal Behavior Processes, 33, 392-408.

Kyonka, E. G. E., \& Grace, R. C. (2008). Rapid acquisition of preference in concurrent chains when alternatives differ on multiple di- mensions of reinforcement. Journal of the Experimental Analysis of Behavior, 89, 49-69.

Kyonka, E. G. E., \& Grace, R. C. (2009). Effects of unpredictable changes in initial-link duration on choice and timing. Behavioural Processes, 81, 227-232.

Mackintosh, N. J., McGonigle, B., Holgate, V., \& Vanderver, V. (1968). Factors underlying improvement in serial reversal learning. Canadian Journal of Psychology, 22, 85-95.

MarK, T. A., \& Gallistel, C. R. (1994). Kinetics of matching. Journal of Experimental Psychology: Animal Behavior Processes, 20, 79-95.

MAZUR, J. E. (1995). Development of preference and spontaneous recovery in choice behavior with concurrent variable-interval schedules. Animal Learning \& Behavior, 23, 93-103.

MazUR, J. E. (1996). Past experience, recency, and spontaneous recovery in choice behavior. Animal Learning \& Behavior, 24, 1-10.

MAZUR, J. E. (1997). Effects of rate of reinforcement and rate of change on choice behavior in transition. Quarterly Journal of Experimental Psychology, 50B, 111-128.

Ploog, B. O. (2001). Effects of primary reinforcement on pigeons' initial-link responding under a concurrent-chains schedule with nondifferential terminal links. Journal of the Experimental Analysis of Behavior, 76, 75-94.

Roberts, W. A., \& Kraemer, P. J. (1982). Some observations of the effect of intertrial interval and delay on delayed matching-to-sample in pigeons. Journal of Experimental Psychology: Animal Behavior Processes, 8, 342-353.

SCHOFIELD, G., \& Davison, M. (1997). Nonstable concurrent choice in pigeons. Journal of the Experimental Analysis of Behavior, 68, 219232

WiLliams, B. A. (1971). The effects of intertrial interval on discrimination reversal learning in the pigeon. Psychonomic Science, 23, 241243.

WiLliams, B. A. (1976). Short-term retention of response outcome as a determinant of serial reversal learning. Learning \& Motivation, 7 , $418-430$

WiLliams, B. A. (1977). Contrast effects in simultaneous discrimination learning. Animal Learning \& Behavior, 5, 47-50.

Williams, B. A. (1998). Relative time and delay of reinforcement. Learning \& Motivation, 29, 236-248.

Williams, B. A., \& FANTino, E. (1978). Effects on choice of reinforcement delay and conditioned reinforcement. Journal of the Experimental Analysis of Behavior, 29, 77-86.

Woodward, W. T., Schoel, W. M., \& BitTerman, M. E. (1971). Reversal learning with singly presented stimuli in pigeons and goldfish. Journal of Comparative \& Physiological Psychology, 76, 460-467.

(Manuscript received December 7, 2008; revision accepted for publication September 20, 2009.) 